\title{
LE LIEU UNIQUE CHOISI PAR YHWH ET LA PLURALITÉ DES TEMPLES DANS L'IDÉOLOGIE DEUTÉRONOMISTE
}

\author{
Thomas RöMER \\ Université de Lausanne - Collège de France \\ thomas.romer@unil.ch
}

\section{Summary}

According to the deuteronomistic tradition there is only one legitimate place to offer sacrifices to $Y H W H$, the Jerusalemite temple (see Dt 12). However, the Deuteronomists were aware that there were other yahwistic cult place, and invented for those they considered to be legitimate (Shilo) the idea of a succession of chosen places (Jer 7). Those they considered to be illegitimate (Bethel) were presented as idolatrous (1 Kgs 12). In the Pentateuch, which is also a compromise between priestly and deuteronomistic ideologies, Lev 17 tries to harmonize between the diversity of yabwistic sanctuaries and the deuteronomistic idea of cult centralization.

\section{Résumé}

Dans la tradition deutéronomiste, Jérusalem est le seul lieu légitime pour offrir des sacrifices à YHWH, comme le montre notamment Dt 12. Néanmoins, les rédacteurs deutéronomistes savent qu'il $y$ avait d'autres sanctuaires yahwistes. Pour ceux qu'ils considèrent légitimes (Shilo), ils inventent l'idée d'une succession de «lieux uniques » (Jr 7); d'autres comme Béthel, sont condamnés comme «idolâtres» (1 R 12). Le Pentateuque qui résulte en grande partie d'un accord entre l'idéologie sacerdotale et l'idéologie deutéronomiste, propose en Lv 17 un compromis entre l'idée de lieu unique et la diversité des sanctuaires yahwistes. 
Selon Otto Kaiser, l'idéologie deutéronomiste se caractérise par l'idée de l'exclusivité: loyauté absolue envers YHWH et envers son seul sanctuaire, le temple de Jérusalem ${ }^{1}$. On trouve, en effet, dans le Deutéronome et dans les livres des Rois une forte insistance sur l'«alliance », c'est-à-dire sur le lien exclusif qui unit YHWH et Israël et, en même temps, un rejet quasi constant de tout lieu de culte, yahwiste ou non yahwiste, en dehors de Jérusalem.

Dans le Pentateuque, l'idée que YHWH se choisira un seul lieu de culte opère une césure entre les livres allant de Genèse à Nombres et le Deutéronome. Le thème du sanctuaire unique pour YHWH rattache le Deutéronome aux préoccupations des livres des Rois. Alors que les récits patriarcaux ou le code d'Alliance ne semblent guère préoccupés par l'idée d'un sanctuaire unique (Jacob est le fondateur du sanctuaire de Béthel et Abraham construit un certain nombre d'autels ${ }^{2}$ ), le leitmotiv des livres des Rois est la fidélité ou l'infidélité des différents rois vis-à-vis du temple de Jérusalem. Le péché impardonnable du royaume d'Israël n'est pas en premier lieu le culte de Baal ou la vénération d'Ashérah (ces choses existent aussi dans le royaume de Juda), mais c'est la construction de temples yahwistes à Béthel et à Dan. Cette insistance sur le seul temple légitime de YHWH peut néanmoins paraître étonnante dans la mesure où la finale des Rois (2 R 24-25) relate la destruction de ce temple. De même, le livre de Jérémie, qui d'une manière ou d'une autre a connu des révisions de type deutéronomiste, comporte une finale similaire à 2 R 24-25 (dans les deux versions du livre, LXX et TM) et même des oracles attribués à Jérémie annonçant la destruction du temple de Jérusalem.

La question qui se pose alors est triple: (1) quelle est la fonction de l'idéologie deutéronomiste de la centralisation du culte et du sanctuaire unique? (2) comment le milieu deutéronomiste explique-t-il l'existence d'autres sanctuaires yahwistes en dehors de Jérusalem ? (3) comment cette idéologie du lieu unique est-elle intégrée dans le Pentateuque?

1. O. KAISER, Einleitung in das Alte Testament: eine Einführung in ihre Ergebnisse und Probleme, Gütersloh, 1984, p. 165-166.

2. Cependant, on a souvent observé que le seul endroit où Abraham offre explicitement un sacrifice (Gn 22), la montagne de Moriah, comporte sans doute une allusion à Jérusalem. 
Je ne rouvrirai pas le dossier sur l'origine et l'évolution de «l'école deutéronomiste ». J'ai exposé ma vision des choses ailleurs et je reste convaincu qu'on ne peut expliquer l'origine du milieu deutéronomiste sans référence à l'époque néo-assyrienne qui me paraît le contexte historique le plus plausible pour la naissance du style et de l'idéologie deutéronomiste ${ }^{3}$. J'aimerais plutôt montrer, dans la suite, comment l'idée de la centralisation, qui probablement a vu le jour au VII ${ }^{e}$ siècle avant l'ère chrétienne, a dû tenir compte d'une multitude de sanctuaires yahwistes depuis le VII ${ }^{e}$ siècle jusqu'à l'époque perse, durant laquelle a eu lieu la dernière grande rédaction deutéronomiste d'un certain nombre de livres (notamment Dt à Rois et Jr).

\section{Le discours sur le sanctuaire unique en Dt 12}

Dt 12 contient une série de répétitions et de réorientations qui font apparaître une complexité dont il s'agit de tenir compte. Si l'on part de la quadruple insistance sur le seul lieu choisi par YHWH, on constate que les trois premiers énoncés se trouvent au centre de trois unités construites de manière parallèle. Dans les trois cas, le commandement principal est précédé d'un énoncé négatif (v. 2-4 [4] : ne pas agir vis-à-vis de YHWH à la manière des nations; v. 8-10 [8]: ne pas agir comme aujourd'hui; v. 13: se garder d'offrir les holocaustes n'importe où); et chaque fois, on trouve en finale un appel à la joie (v. 7, v. 12, v. 18) $)^{4}$. Ces observations permettent de délimiter trois unités comme suit: 2-7 (employant le «vous »), 8-12 (en «vous »), 13-18 [195] (en «tu »). La première péricope a un lien évident avec les v. 29-31, tandis que l'unité 13-18 est reprise en 20-28.

3. T. RömER, La première histoire d'Israël. L'École deutéronomiste à l'auvre, Genève, 2007, p. 51-114.

4. Voir R. Smend, Die Entstehung des Alten Testaments, Stuttgart, 1978. p. $72-73$.

5. L'insistance sur le lévite au v. 19 pourrait provenir d'une rédaction tardive dans l'esprit des Chroniques, voir U. DAHMEN, Leviten und Priester im Deuteronomium. Literakritische und redaktionsgeschichtliche Studien, Bodenheim, 1996, p. 379. 


\begin{tabular}{llll}
\hline & Vous & Vous & Tu \\
\hline Enoncé négatif & $2-4$ & $8-10$ & 13 \\
& ne pas agir & ne pas agir & ne pas offrir \\
& comme les & comme «main- & des sacrifices \\
& nations & tenant» & n'importe où \\
Loi de centralisation & $5-6$ & 11 & 14 \\
Appel à la joie & 7 & 12 & 18 \\
\hline
\end{tabular}

III

II

I

Ce découpage qui rejoint un certain consensus exégétique ${ }^{6}$ permet de distinguer (au moins) trois époques et trois théologies différentes concernant le sanctuaire unique. Il est généralement reconnu qu'à l'intérieur de Dt 12,1-19, les v. 13-18 constituent le noyau auquel sont venus s'ajouter d'abord les versets 8-12, puis 2-7.

On a souvent fait observer que l'hypothèse d'une succession chronologique des trois versions de la loi de centralisation est confirmée par le développement de la formule de centralisation qui, du v. 14 au v. 5, en passant par le v. 11, s'allonge de plus en plus. Dans la forme «finale »du chapitre qui s'est constituée à l'époque perse, le lecteur/auditeur passe en revanche de la forme la plus longue à la forme la plus courte:

v. 5: Le lieu (maqom) que YHWH, votre Dieu, choisira parmi toutes $(k o l)$ vos tribus pour y mettre son nom, pour le faire habiter $(s k n)^{7}$.

v. 11 : Le lieu (maqom) que YHWH, votre Dieu, choisira pour y faire habiter (rakken) son nom.

6. Voir E. Reuter, Kultzentralisation. Entstehung und Theologie von Dtn 12, Francfort, 1993, p. 100-114; E. Nielsen, Deuteronomium, Tübingen, 1995, p. 135-136; M. KELLER, Untersuchungen zur deuteronomisch-deuteronomistischen Namenstheologie, Weinheim, 1996, p. 25-44; B. M. Levinson, Deuteronomy and the Hermeneutics of Legal Innovation, New York-Oxford, 1997, p. 21-28; U. RÜterswörden, Deuteronomium, Neukirchen-Vluyn, 2011, p. 1-57.

7. La vocalisation massorétique de $l k n w$ dans le texte massorétique pose problème. En mettant la césure principale après šam («là-bas »), les Massorètes ont compris škn comme l'objet du verbe suivant. Dans le texte pré-massorétique $l s k k n w$ était compris comme infinitif pi'el et finale de la formule de centralisation, voir les arguments présentés par M. KELLER, Untersuchungen zur deuteronomisch-deuteronomistischen Namenstheologie, Weinheim, 1996, p. 15-17. 
v. 14: Le lieu (maqom) que YHWH choisira dans une ('èhad) de tes tribus.

Selon le v. 14, YHWH choisira son lieu dans une seule tribu, ce qui s'oppose à l'interprétation selon laquelle les v. 13-14 signifieraient que YHWH choisira différents sanctuaires en même temps ou d'une manière successive ${ }^{8}$. Le באחד שבטיך renvoie certainement au יהוה אחד de Dt 6,4, et cela davantage encore si Dt $12,13 \mathrm{ss}$ a été, à l'origine, conçu comme suite directe de Dt 6,4-5:

«Écoute Israël, YHWH notre Dieu, est YHWH UN ('éhad). Tu aimeras YHWH ton Dieu de tout (be-kol) ton cœur, de tout $(b e-k o l)$ ton être, de toute $(b e-k o l)$ ta force. Garde-toi de faire monter tes holocaustes en tout $(b e-k o l)$ lieu que tu vois. Seulement en ce lieu que YHWH choisira dans UNE ('èbad) de tes tribus, c'est là que tu feras monter tes holocaustes et là tu feras tout $(k o l)$ ce que je te commande ».

Au Dieu unique auquel il faut s'attacher de toute sa personne correspond l'élection du sanctuaire unique dans la tribu unique ainsi que le rejet de la totalité des lieux saints et, sous-entendu, de la totalité des autres tribus (c'est-à-dire du royaume du Nord). Il ne fait guère de doute que la tribu unique selon Dt 12,13-14 est la tribu de Juda, et le lieu unique le temple de Jérusalem.

La même idée sous-tend peut-être la partie la plus ancienne de la prière de Salomon lors de l'inauguration du temple en 1 $\mathrm{R} 8,16$, un passage qui pose cependant de nombreux problèmes de critique textuelle. Si l'on suit la LXX (qui, en Rois, se fonde souvent sur un texte hébreu plus ancien que le TM), on peut reconstruire le texte primitif comme suit:

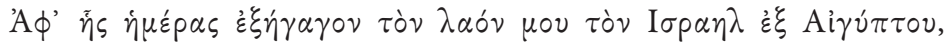

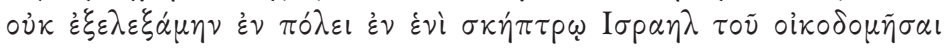

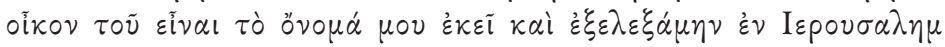

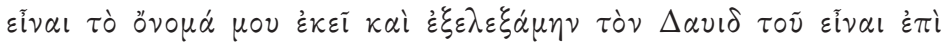

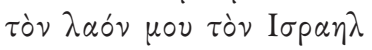

8. E. A. Knauf, «The Glorious Days of Manasseh », dans L. L. GRaBBE (éd.), Good Kings and Bad Kings, Londres-New York, 2005, p. 164-188, spécialement p. 178. Pour une critique, voir K. L. NolL, «Deuteronomistic History or Deuteronomistic Debate? (A Thought Experiment)», Journal for the Study of the Old Testament 33 (2007), p. 311-345, spécialement p. 331, n. 58, et U. RÜTERSwörden, Deuteronomium, NeukirchenVluyn, 2011, p. 38. 


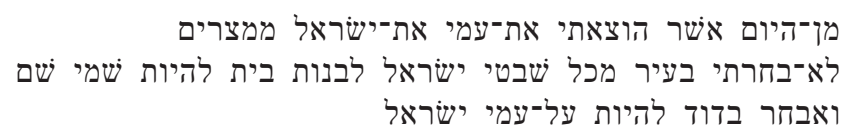

(Texte reconstruit): Depuis le jour où j'ai fait sortir mon peuple d'Égypte, je n'ai pas choisi de ville dans une tribu pour construire une maison ${ }^{9}$ pour que mon nom y soit, et j'ai choisi Jérusalem pour que mon nom y soit et j'ai choisi David pour qu'il soit sur mon peuple Israël.

Si Dt 12,14 vise donc la légitimation de la seule tribu de Juda, pourquoi alors la version plus récente de la formule de l'élection du lieu en Dt 12,5 parle-t-elle de «toutes les tribus »?

כי אם־אל־המקום אשר־יבחר יהוה אלהיכם מכל־שבטיכם לשום את־שמו

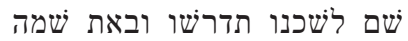

Ce changement de l'expression de Dt 12,14 s'explique, me semble-t-il, par un problème né au moment de la construction d'une «histoire deutéronomiste » qui englobe (au moins) les livres allant du Deutéronome jusqu'aux Rois. Au moment où le Deutéronome est conçu comme grille de lecture d'une histoire qui se déploie dans les livres des Prophètes antérieurs, le commandement du sanctuaire unique se trouve confronté à l'existence d'autres sanctuaires yahwistes. Pour résoudre ce problème, les Deutéronomistes élaborent différentes stratégies: la dissimulation, le dénigrement, la théorie d'une succession de sanctuaires, la caricature.

\section{La dissimulation de sanctuaires dans l'histoire deutéronomiste}

L'archéologie et la Bible hébraïque indiquent qu'il existait un nombre important de sanctuaires yahwistes qui ne sont pas mentionnés dans l'histoire deutéronomiste. Ainsi les textes de Kuntillet Ajrud mentionnent un YHWH de Téman et un YHWH de Samarie:

9. Le pluriel du TM מכל שבטי ישראל peut s'expliquer comme une harmonisation avec Dt 12,5 (on y reviendra). La mention de l'élection de Jérusalem qui est attestée dans le texte parallèle de 2 Ch 6,5 a probablement disparu du TM suite à un homoioarkton, voir les commentaires. 
Pithos A. 1: 1. Dit '[...] (NP 1)...: « Dis à Yehalle'[lel ?] (NP 2), Yoasa (NP 3) et... (NP 4?): Je vous bénis (ou: je vous ai bénis) 2. par YHWH de Samarie et son Ashérah.

Pithos B. 2: 5-8. Je te bénis (ou: je t’ai béni) par YHWH de Téman et par son Ashérah. Qu'il (= YHWH) (te) bénisse et te garde (une formule quasiment identique se trouve en Pithos B. 3).

La mention d'un YHWH de Téman (probablement en territoire édomite) indique clairement qu'il existait des sanctuaires yahwistes en dehors d'Israël et de Juda.

La meilleure façon de comprendre l'invocation d'un YHWH de Samarie est de penser qu'il existait un temple de YHWH dans la capitale d'Israël ${ }^{10}$. Dans ce sens, on peut rappeler le prisme de Nimrud qui, en relatant la destruction de Samarie, évoque la déportation des «dieux en lesquels ils se confiaient». Le livre d'Osée contient des critiques contre le «veau de Samarie», comme par exemple en Os 8,5-6:

5 «Il a rejeté ton veau, Samarie! Je suis en colère contre eux. Jusqu'à quand seront-ils incapables de parvenir à la pureté ? ${ }^{6}$ Car il provient d'Israël, un artisan l'a fait, ce n'est pas un dieu! Oui, le veau de Samarie sera mis en pièces ${ }^{11} \gg$.

Il paraît en effet plausible qu'Omri ait fait construire un sanctuaire pour YHWH dans la capitale, ce qui n'exclut pas que Béthel ait été le plus important sanctuaire yahwiste du Nord, comme le texte d'Amos 7,10-17 semble l'indiquer. Mais les rédacteurs deutéronomistes n'en parlent pas directement.

Au sujet du roi Akhab, on trouve en $1 \mathrm{R}$ 16,32 la notice suivante:

ויקם מזבח לבעל בית הבעל אשר בנה בשמרון

10. La solution alternative serait de comprendre Samarie comme le nom d'un territoire et non de la ville mais, comme le montrent par exemple les parallèles néo-assyriens (Ishtar d'Arbèle) ou du Levant (Hadad de Sikan dans l'inscription de Tell Fekherye), il s'agit plutôt de temples où la divinité se manifeste.

11. Un texte plus ancien a été retravaillé dans le sens de la polémique contre les images que l'on trouve dans le Second Ésaïe. L'oracle (oséen ?) primitif exprime une critique contre la statue de Samarie, sans polémique en général sur les représentations du divin; H. Pfeıffer, Das Heiligtum von Bethel im Spiegel des Hoseabuches, Göttingen, 1999. 
Il éleva un autel pour Baal dans la maison de Baal qu'il (= Omri ?) avait bâtie.

Le redoublement de Baal est un peu curieux : pourquoi préciser que le roi installe un autel pour Baal dans le temple de Baal? Apparemment nous avons ici affaire à une altération du texte original qui parle d'un beyt 'elohim ${ }^{12}$ ou d'un beyt $Y H W H$ :

Il éleva un autel pour Baal dans la maison de YHWH (dans la maison de Dieu) qu'il avait bâtie.

On aurait alors ici l'indication que le roi Omri - que les rédacteurs bibliques abhorrent - aurait construit dans sa nouvelle capitale un temple pour YHWH, à l'intérieur duquel il aurait également prévu des installations pour une vénération d'autres dieux.

Le passage de 2 R 10,21-27 relate la destruction par Jéhu d'un temple de Baal à Samarie. Peut-être les rédacteurs deutéronomistes voulaient-ils faire passer un sanctuaire yahwiste pour un sanctuaire dédié à Baal. À en croire les graffiti de Kuntillet Ajrud, YHWH avait un sanctuaire dans cette ville. Le dossier archéologique ne permet cependant pas (encore) de résoudre définitivement la question d'un sanctuaire yahwiste à Samarie ${ }^{13}$.

La stèle de Mésha, quant à elle, indique l'existence d'un sanctuaire yahwiste au $\mathrm{IX}^{\mathrm{e}}$ siècle en Transjordanie. On y lit entre autres :

«Et Kamosh me dit: 'Va, prends Nebo à Israël'. J'allai de nuit et je l'attaquai depuis le lever du jour jusqu'à midi. Je la pris et je tuai tout, à savoir sept mille hommes et garçons, femmes, filles et concubines parce que je les avais voués ( $\mathrm{brm}$ ) à 'Ashtar de Kamosh. J'emportai de là les vases de YHWH et je les traînai devant la face de Kamosh ».

Le mot traduit par «vases» $(k l y)$ est assez général et peut désigner toutes sortes d'objets cultuels, peut-être même la statue. De toute façon, cette remarque présuppose un sanctuaire de $Y H W H$ à Nebo que Mésha aurait détruit et dont il aurait dé-

12. S. Tiмm, Die Dynastie Omri: Quellen und Untersuchungen zur Geschichte Israels im 9. Jahrhundert vor Christus, Göttingen, 1982, p. 3233.

13. D. JERICKe, Regionaler Kult und lokaler Kult: Studien zur Kultund Religionsgeschichte Israels und Judas im 9. und 8. Jahrbundert v. Chr, Wiesbaden, 2010, p. 90-91. Il n'y a pas d'indice en faveur d'un culte royal. 
placé, comme c'est la coutume, les ustensiles ou statues dans le temple de Kemosh.

Il y avait sans doute également un temple de YHWH à Lakish, étant donné que les reliefs assyriens mettant en scène la destruction de la ville font apparaître un groupe de soldats emportant des objets cultuels de grande taille qui présupposent l'existence d'un sanctuaire dont les livres des Rois et de Jérémie, qui mentionnent la ville à plusieurs reprises, ne disent rien.

Dans le Sud, on peut encore mentionner le sanctuaire d'Arad qui probablement fut un temple dédié à YHWH et qui, pour des raisons peu claires, fut «fermé » durant le $\mathrm{VII}^{\mathrm{e}}$ siècle $^{14}$. Un tel sanctuaire n'est jamais mentionné dans des textes bibliques.

Le silence des rédacteurs deutéronomistes concerne des sanctuaires qui étaient en fonction durant l'époque des monarchies israélite et judéenne. La discrétion quant aux sanctuaires judéens en dehors de Jérusalem peut s'expliquer par le fait qu'on ne voulait pas admettre leur existence après la construction du temple de Jérusalem. Peut-être sous-entendait-on leur existence via les bamôt, des sanctuaires à ciel ouvert - l'existence desquels est fréquemment dénoncée.

Pour le royaume d'Israël, les rédacteurs deutéronomistes construisent le sanctuaire de Béthel (avec celui de Dan) comme étant la manifestation du «péché de Jéroboam ». Le fait qu'ils ne critiquent pas directement le temple de Samarie s'explique peut-être par la popularité de «YHWH de Samarie » attestée dans les inscriptions de Kuntillet Ajrud.

\section{Le dénigrement des sanctuaires du «Nord»}

Selon $1 \mathrm{R} 12$, Jéroboam fit construire deux sanctuaires à Béthel et à Dan où il érigea des statues bovines, qu'il identifia comme représentant le dieu qui a fait sortir Israël d'Égypte:

${ }^{28}$ Le roi Jéroboam prit conseil et fit deux veaux d'or et dit au peuple: 'Vous êtes trop souvent montés à Jérusalem; voici tes dieux, Israël, qui t'ont fait monter du pays d'Egypte'. ${ }^{29}$ Il plaça

14. D. Ussishinin, «The Date of the Judaean Shrine at Arad», Israel Exploration Journal 38 (1988), p. 142-157; Z.E. Herzog, « The Date of the Temple at Arad: Reassessment of the Stratigraphy and the Implications for the History of Religion in Judah », dans A. MAzAR (ed.), Studies in the Archaeology of the Iron Age in Israel and Jordan, Sheffield, 2001, p. 156-178. 
l'un à Béthel, et l'autre, il l'installa à Dan ${ }^{30}$ - c'est en cela que consista le péché. Le peuple marcha en procession devant l'un [des veaux] jusqu'à Dan.

L'installation des statues à Béthel et à Dan symbolise les frontières septentrionale et méridionale du royaume. Concernant l'archéologie, la mention de Dan dans le contexte de la fin du $\mathrm{X}^{\mathrm{e}}$ siècle pose problème, car il semble que Dan n'ait été israélite que depuis le VIII ${ }^{e}$ siècle ${ }^{15}$. Dans ce cas, la fondation d'un sanctuaire à Dan pourrait être une rétroprojection de l'époque de Jéroboam II; on pourrait même se demander si l'ensemble du récit de $1 \mathrm{R} 12$ n'est pas une rétroprojection du temps de Jéroboam II vers Jéroboam I, dans le but de mettre en scène le «péché originel» du royaume d'Israël.

Le pluriel dans l'exclamation de Jéroboam («tes dieux ») est étonnant; il est d'ailleurs repris dans l'histoire du veau d'or en Exode 32 qui transfère le «péché de Jéroboam» au Sinaï. Le pluriel pourrait s'expliquer par le fait qu'il s'agit de deux statues. Ex 32 serait alors seulement une citation de l'énoncé de Jéroboam.

\begin{tabular}{|c|c|}
\hline $1 \mathrm{R} 12,28$ & Ex 32,4 \\
\hline הנה אלה מצרים ישראל אשר העלוך & מארז אלהיך ישראל אשר העלוך \\
\hline $\begin{array}{l}\text { Voici tes dieux, Israël, qui t'ont fait } \\
\text { monter du pays d'Égypte. }\end{array}$ & $\begin{array}{l}\text { Ce sont tes dieux, Israël, qui t'ont } \\
\text { fait monter du pays d'Égypte. }\end{array}$ \\
\hline
\end{tabular}

Une autre hypothèse serait d'imaginer qu'il s'agit d'un couple divin : tous les deux trônaient sur des piédestaux thériomorphes ${ }^{16}$. Il pourrait donc être question d'une parèdre de YHWH. Mais cela reste assez spéculatif et il n'y a aucune attestation d'une association d'Ashérah ou d'une autre déesse à l'Exode. Peut-être le pluriel reflète-t-il simplement la stratégie deutéronomiste de présenter le culte yahwiste d'Israël comme un culte «polythéiste ${ }^{17}$.

15. E. Arie, «Reconsidering the Iron Age II Strata at Tel Dan: Archaeological and Historical Implications », Tel Aviv 35 (2008), p. 6-64.

16. E.A. KNAuF, «Bethel », Religion in Geschichte und Gegenwart 1 (1998), col. 1375-1376.

17. Selon M. Noтн, 1 Könige 1-16, Neukirchen-Vluyn, 1968, p. 282, le pluriel de la forme verbale interprète le culte de Dan et Béthel: «im Sinne von Vielgötterei ». 
Le livre d'Osée indique la vénération de YHWH sous la forme d'un taureau à Samarie ${ }^{18}$. Le texte de $1 \mathrm{R} 12$ pourrait alors constituer un transfert du taureau de Samarie à Béthel. Il est cependant également possible qu'ait existé un culte bovin à Béthel ${ }^{19}$.

Dans la construction deutéronomiste, l'action de Jéroboam en $1 \mathrm{R} 12$ ouvre le thème du «péché de Jéroboam » à la suite duquel la plupart des rois du $\operatorname{Nord}^{20}$ sont blâmés, et qui mena selon $2 \mathrm{R} 17$ à la chute du royaume d'Israël et de sa capitale Samarie. Tous les Israélites sont dits avoir « continué dans tous les péchés qu'avait commis Jéroboam; ils ne s'en écartèrent pas » (2 R 17,22). Le «péché de Jéroboam» sera ensuite aboli par le roi Josias: «l'autel de Béthel, le haut lieu construit par Jéroboam, fils de Nebat, qui avait fait pécher Israël - cet autel et ce haut lieu, il les démolit» $(2 \mathrm{R} 23,15)$. Or, l'affirmation de la destruction du sanctuaire - qui ne correspond pas à la réalité historique - a nécessité plus tard une autre appréciation du sanctuaire de Béthel ${ }^{21}$ qui a joué un rôle majeur après la destruction du temple de Jérusalem. Nous y reviendrons.

\section{La théorie d'une succession de sanctuaires}

Dans le cadre des Prophètes antérieurs, Shilo apparaît pour la première fois en Jos $18,1.8-10 ; 19,51 ; 21,2 ; 22,9.12$. Selon Jos 18,1, les Israélites, après la prise de possession du pays, y érigèrent le premier sanctuaire, le אהל מועד. Il ne s'agit pas d'un

18. Dans un ostracon de Samarie, on trouve un nom propre 'glyw (Samaria 41), qu'on peut traduire par « veau de $\mathrm{YHWH}$ » ou par « YHWH est un veau ».

19. E. Würthwein, Die Bücher der Könige, 1 Könige 1-16, Göttingen, 1977, p. 164.

20. Un changement intéressant se produit dans les formules d'évaluation. Les successeurs de Jéroboam, de Nadab à Joram, le dernier Omride, sont fondamentalement regardés de la même façon : ils imitent Jéroboam et/ou ses fautes, provoquant ainsi la colère de YHWH. À partir de Jéhu jusqu'à Peqah, la même formule caractérise chaque roi : «il fit ce qui est mal aux yeux de YHWH, il ne s'écarta pas des péchés de Jéroboam, fils de Nebat, qui fit pécher Israël ». Le dernier roi, Osée, «fit le mal aux yeux de YHWH mais pas comme les rois d'Israël qui l'ont précédé » (2 R 17,2). Les rédacteurs deutéronomistes voulaient donc faire une différence entre les premiers neuf rois et les neuf rois suivants.

21. Un sanctuaire yahwiste à Dan est très rarement mentionné, à l'exception de $1 \mathrm{R} 12$. Il apparaît encore (comme glose ?) dans le renvoi au péché de Jéroboam en 2 R 10,29, et dans le texte obscur d'Am 8,14. 
texte de facture deutéronomiste, mais d'un passage plus récent qui reprend l'idée sacerdotale de la «tente de la rencontre». En Jos 22,29, lorsque les tribus transjordaniennes renoncent à la construction d'un autel, elles affirment que le seul autel légitime se trouve devant le mishkan (מזבח יהוה אלהינו אשר לפני משכנ) 1), sans doute également une allusion à Shilo22. Shilo réapparaît ensuite en Jg 18-21, souvent considéré comme un appendice à l'édition deutéronomiste du livre des Juges. En Jg 18,31, il est question d'une époque où le temple de Dieu se trouvait à Shilo: כל־ימי היות האלהים בית בשלה. Apparemment, ces textes tardifs gardent le souvenir d'un sanctuaire yahwiste dans cette localité en Ephraïm dont l'importance à l'époque du passage de l'âge du Bronze récent à l'âge du Fer est confirmée par l'archéologie:

The important role of Shiloh is reflected in the density of the Iron I sites around it... Since a considerable part of it (= the settlement) was probably occupied by the shrine complex and other public buildings, the possibility that Shilo was primarily a sacred temenos... should not be ruled out ${ }^{23}$.

Dans le cadre de l'édition deutéronomiste du livre de Samuel, le sanctuaire de Shilo joue un rôle important et positif. C'est là que se situe la légende de la naissance de Samuel. 1 S 1 présuppose un sanctuaire yahwiste vers lequel on fait des pèlerinages, et $1 \mathrm{~S}$ 3,1 relate sans critique que YHWH se révèle à Samuel à Shilo.

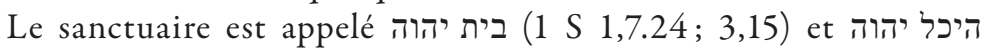
(1 S 1,9; 3,3). Comme le dit Jürg Hutzli : «Der Erzähler vermittelt die Vorstellung eines Gotteshauses mit festen Mauern $»^{24}$. Shilo est également le lieu où se trouve l'arche de YHWH que David fit déménager plus tard à Jérusalem (2 S 6). Ensuite, Shilo disparaît de l'histoire deutéronomiste; il est encore une fois mentionné en $1 \mathrm{R} 14$ comme lieu de rattachement du prophète Ahiyah. Rien de négatif n'apparaît au sujet de Shilo, la seule exception concerne l'oracle contre la maison d'Eli, un ajout de l'époque perse qui reflète un conflit entre les lévites (les Elides)

22. T. C. Butler, Joshua, Waco/Texas, 1983, p. 249-250.

23. I. Finkelstein, «Seilun, Khirbet», dans D. N. Freedman (éd.), Anchor Bible Dictionary, V, New York, 1992, p. 1069-1072, spécialement p. 1072.

24. J. Hutzli, Die Erzählung von Hanna und Samuel: Textkritische und literarische Analyse von 1. Samuel 1-2 unter Berücksichtigung des Kontextes, Zürich, 2007, p. 213. 
et les Sadocides ${ }^{25}$. La vision neutre, voire positive, du temple de Shilo s'explique par la reprise d'une tradition ancienne qui est sans doute liée à un souvenir historique. À un moment donné se posa pourtant la question de la compatibilité de la tradition de Shilo avec l'idée de l'élection du lieu unique en une seule tribu (Dt 12,14).

S'élabora alors une théorie d'une succession des lieux uniques choisis. Dans la dernière révision de Dt 12 , il est maintenant question d'un choix de YHWH à partir de toutes les tribus, ce qui inclut les tribus du Nord permettant une lecture chronologique de l'idée du sanctuaire unique. Une autre tentative de corréler Shilo et le temple de Jérusalem se trouve peut-être en $2 S 7,6$ :

כי לא ישבתי בבית למיום העלתי את־בני ישראל ממצרים ועד היום הזה ואהיה מתהלך באהל ובמית למיום העלת

Souvent ce verset est considéré dans sa forme actuelle comme la révision d'un oracle antérieur. Peut-être le משכן fait-il allusion à Shilo ${ }^{26}$ ? Dans ce cas, le texte suggèrerait une sorte de compromis entre le sanctuaire «provisoire» de Shilo (et peut-être d'autres) et le בית de Jérusalem²

Cependant la théorisation explicite de la relation entre Shilo et le temple de Jérusalem se trouve à l'extérieur de l'histoire deutéronomiste, dans le rouleau deutéronomiste de Jérémie, dans le discours du temple en $\mathrm{Jr} 7^{28}$. Nous ne pouvons reprendre ici la discussion sur la formation de ce chapitre. Dans sa forme actuelle, Jr 7 est construit pour faire écho au chapitre 26 qui relate les conséquences du discours sur le temple. L'annonce de la destruction du temple est ici expliquée par le comportement inadéquat des destinataires et il est mis en parallèle avec l'abandon du sanctuaire de Shilo:

25. Voir l'analyse détaillée de J. Hutzli, Die Erzählung von Hanna und Samuel: Textkritische und literarische Analyse von 1. Samuel 1-2 unter Berücksichtigung des Kontextes, Zürich, 2007, p. 167-202, 268-269.

26. Voir Jos 22,19.

27. Voir dans ce sens B. Halpern, «Shiloh», dans D. N. FreedMAN (éd.), Anchor Bible Dictionary, V, New York, 1992, p. 1213-1215, spécialement p. 1214.

28. Dans la LXX, la localisation n'est pas explicite, mais le contenu suggère un discours tenu dans le temple ou au moins traitant du sort du temple de Jérusalem. 
${ }^{8}$ Mais voici que vous vous fiez à des paroles mensongères, qui ne profiteront pas. ${ }^{9}$ Quoi! Voler, tuer, commettre l'adultère, se parjurer, encenser Baal, suivre des dieux étrangers que vous ne connaissez pas, ${ }^{10}$ puis venir se présenter devant moi en cette maison sur laquelle mon nom est appelé, et dire: «nous voilà sauvés » pour continuer toutes ces abominations! ${ }^{11} \mathrm{~A}$ vos yeux, est-ce devenu un repaire de brigands, cette maison sur laquelle mon nom est appelé ? Moi, en tout cas, je vois, oracle de YHWH! ${ }^{12}$ Allez donc au lieu qui fut le mien, à Shilo où je fis habiter mon Nom auparavant; regardez ce que je lui ai fait, à cause de la perversité de mon peuple Israël. ${ }^{13}$ Et maintenant, puisque vous avez commis tous ces actes oracle de YHWH puisque vous n'avez pas écouté quand je vous parlais instamment et sans me lasser, et que je vous ai appelés et vous n'avez pas répondu, ${ }^{14}$ je vais traiter cette maison sur laquelle mon Nom est appelé, et dans laquelle vous placez votre confiance, ce lieu que j'ai donné à vous et à vos pères, comme j'ai traité Shilo.

Le texte de Jr 26 se réfère au même discours et le résume de la manière suivante:

${ }^{4}$ (...) Ainsi parle YHWH: si vous ne m'écoutez pas pour suivre ma loi que j'ai mise devant vous, ${ }^{5}$ pour écouter les paroles de mes serviteurs, les prophètes, que je vous envoie, que je vous ai envoyés, inlassablement, et que vous n'avez pas écoutés, ${ }^{6}$ alors je traiterai cette maison comme Shilo et je ferai de la [LXX; TM: cette] ville un objet de malédiction pour toutes les nations de la terre.

Les deux textes présentent Shilo comme prédécesseur de Jérusalem en reprenant et en modifiant le vocabulaire deutéronomiste du Nom de Dt 12, 1 R 8 et d'autres textes. Les deux textes suggèrent une destruction de Shilo, bien que celle-ci ne soit nullement relatée dans les textes bibliques. On discute pour savoir s'il s'agit d'une destruction du début du premier millénaire ou de l'époque néo-assyrienne. Concernant l'archéologie, Shilo ne fut pas une localité importante à l'époque des Assyriens. De toute façon, selon la pensée deutéronomiste, cette présumée destruction a dû intervenir avant la construction du temple ${ }^{29}$. Moins que la destruction, c'est l'idée que YHWH a choisi un

29. J. DAY, «The Destruction of the Shiloh Sanctuary and Jeremiah vii, 12,14 », dans J. A. EMERTON (ÉD.), Studies in the Historical Books of the Old Testament, Leyde, 1973, p. 87-94 et 88-89, contra R. A. PEARCE, 
autre sanctuaire (dans le Nord!) qui constitue le point central de cette réflexion, permettant de penser ensemble le lieu unique et la diversité des sanctuaires.

Cette même idée apparaît dans le Psaume 78 qui se trouve également proche de l'idéologie deutéronomiste ${ }^{30}$. Vers la fin de ce psaume, l'auteur oppose au rejet d'Ephraïm, le choix de la tribu de Juda, ce qui correspond à Dt 12,14 et 2 R 17,18:

\begin{tabular}{|l|l|}
\hline $\begin{array}{l}\text { Ps 78,67: Il écarta la famille de } \\
\begin{array}{l}\text { Joseph, } \\
\text { il refusa de choisir la tribu } \\
\text { d'Ephraïm. }\end{array}\end{array}$ & $\begin{array}{l}\text { 2 R 17,18: YHWH s'est mis dans } \\
\text { une violente colère contre Israël; } \\
\text { il les a écartés loin de sa présence. } \\
\text { Seule est restée la tribu de Juda. }\end{array}$ \\
\hline $\begin{array}{l}\text { Ps 78,68: Il choisit la tribu de } \\
\text { Juda, } \\
\text { la montagne de Sion qu'il aime. }\end{array}$ & $\begin{array}{l}\text { Dt 12,14: C'est seulement au lieu } \\
\text { que YHWH choisira, dans l'une } \\
\text { de tes tribus, que tu offriras tes } \\
\text { holocaustes. }\end{array}$ \\
\hline
\end{tabular}

Mais auparavant, le psaume reprend la même idée que Jr 7 et 26 :

${ }^{58}$ Ils l'indignaient avec leurs hauts lieux; leurs idoles excitaient sa jalousie. ${ }^{59}$ Dieu entendit et s'emporta, il rejeta complètement Israël; ; ${ }^{60}$ il quitta la demeure de Shilo, la tente qu'il avait dressée parmi les hommes (ויטש משכן שלו אהל שכן באדם). ${ }^{61}$ Il livra sa force à la captivité, sa majesté à des mains ennemies. ${ }^{62} \mathrm{Il}$ abandonna son peuple à l'épée, il s'emporta contre son patrimoine.

Le psaume considère donc Shilo comme lieu de la présence de YHWH, en reprenant l'idée de Jos 22 et de 2 S 7, selon laquelle il s'agit d'une «demeure » et d'une «tente», et non d'un 《sanctuaire» (מקדש), terme réservé au temple de Jérusalem au v. 69. Le moment où le psalmiste situe le rejet de Shilo n'est pas entièrement clair. Les v. 61-62 pourraient faire penser à la chute de Samarie et les v. 67-69 mettent en contraste le rejet d'Israël et le choix de Sion et David ${ }^{31}$. Le psaume veut peut-être permettre

«Shiloh and Jer. vii 12, $14 \& 15$, Vetus Testamentum 23 (1973), p. 105108.

30. M. Witte, «From Exodus to David - History and Historiography in Psalm $78 \gg$, dans N. Calduch-Benages - J. Liesen (éd.), How Israel's Authors Viewed Its Earlier History. International Conference of the ISDCL at Barcelona, Spain, 2-6 July 2005, Berlin-New York, 2006, p. 21-42.

31. T. Römer, « Extra-Pentateuchal Biblical Evidence for the Existence of a Pentateuch? The Case of the «Historical Summaries», Especially in the Psalms », dans T. B. Dozeman - K. Schmid - B. J. Schwartz (éd.), 
une double lecture: le rejet de Shilo fait pendant à l'élection de David et de Sion; la chute d'Israël est la dernière conséquence de l'abandon de Shilo; le psaume ne parle pas explicitement de destruction. De toute façon, la stratégie déployée en Jr 7;26 et Ps 78 permet ainsi d'accepter la légitimité d'autres sanctuaires yahwistes avant la construction du temple de Jérusalem ${ }^{32}$.

\section{La caricature d'un sanctuaire existant à côté du temple de Jérusalem: le cas de Béthel}

À un certain niveau narratif de l'histoire deutéronomiste, Josias met fin au sanctuaire de Béthel $(2$ R 23,15). Cette affirmation contredit, cependant, la situation du $\mathrm{VI}^{\mathrm{e}}$ siècle où Béthel était apparemment un sanctuaire concurrent du temple de Jérusalem détruit et a dû attirer des pèlerins du Nord et du Sud. L'existence de ce sanctuaire ne pouvait plus être niée. Ainsi des rédacteurs du début de l'époque perse augmentent-ils $2 \mathrm{R} 17$ d'une nouvelle étiologie du sanctuaire de Béthel.

Cet ajout polémique - et sans doute tardif - au récit primitif de 2 R 17 montre que le culte yahwiste continuait en Samarie:

${ }^{24}$ Le roi d'Assyrie fit venir des gens de Babylone, de Kouth, de Awa, de Hamath et de Sefarwaïm et les établit dans les villes de Samarie à la place des fils d'Israël. Ils prirent possession de la Samarie et en habitèrent les villes. ${ }^{25}$ Or, au début de leur installation en ce lieu, comme ils ne craignaient pas YHWH, YHWH envoya contre eux des lions qui les tuèrent. ${ }^{26} \mathrm{Ils} \mathrm{dirent}$ au roi d'Assyrie: «Les nations que tu as déportées et établies dans les villes de Samarie ne connaissent pas la façon d'honorer le dieu du pays. Ce dieu a envoyé contre elles des lions, et voilà que ceux-ci les font mourir car elles ne connaissent pas la façon d'honorer le dieu du pays ». ${ }^{27}$ Le roi d'Assyrie donna cet ordre: «Faites partir là-bas un des prêtres de Samarie que vous avez déportés, qu'il aille habiter là-bas et qu'il leur enseigne la façon d'honorer le dieu du pays $\gg .{ }^{28}$ L'un des prêtres qu'on avait déportés de Samarie vint donc habiter Béthel; il leur enseignait comment on devait craindre YHWH.

The Pentateuch. International Perspectives on Current Research, Tübingen, 2011, p. 471-488, spécialement p. 486-487.

32. Voir aussi le cas de Gabaon en 1 R 3 : la présence de Salomon et la révélation divine ne posent pas de problème. 
${ }^{29}$ En fait, chaque nation se fit son dieu et le plaça dans les maisons des hauts lieux, que les Samaritains avaient construites. Chacune des nations agit ainsi dans les villes où elle résidait : ${ }^{30}$ les gens de Babylone firent Soukkoth-Benoth («huttes de filles»); ceux de Kouth Nergal; ceux de Hamath Ashima; ${ }^{31}$ les Awites, Nibhaz et Tartaq; les Sefarwaïtes brûlèrent leurs fils au feu pour Adrammélek et Anammélek, dieux de Sefarwaïm. ${ }^{32}$ Ils craignirent YHWH et se firent, parmi les leurs, des prêtres de hauts lieux pour officier en leur nom dans les maisons des hauts lieux. ${ }^{33}$ Tout en craignant YHWH, ils servaient leurs propres dieux, selon le rite des nations d'où on les avait déportés ».

Le texte dans sa forme actuelle est tardif, et appartient à l'époque perse, reflétant sans doute des polémiques anti-samaritaines ${ }^{33}$. Le caractère tardif du passage est assuré par le style de l'hébreu: par exemple le verbe «être », combiné avec un participe pour remplacer la forme narrative (wayyiqtol) est typique de l'hébreu postbiblique; on trouve neuf fois cette construction dans les versets 25-41, alors qu'elle est absente du reste du chapitre.

Néanmoins, le texte peut garder quelques souvenirs sur la situation en Samarie après son incorporation dans l'empire assyrien. On apprend que le roi assyrien peuple la Samarie avec des personnes venues de Babylone et peut-être aussi de Syrie ${ }^{34}$. Hamat peut être la ville sur l'Oronte, mais la distance avec Samarie n'est pas grande, ou alors Amati dans le sud de la Mésopotamie ${ }^{35}$. Sefarwaïm est soit Sippar, soit Sipira'ni non loin de Nippur. Cette ville est mentionnée dans les documents de Murashu dans lesquels sont également attestés des noms judéens (ou israélites) à Babylone, à l'époque perse. Apparemment, le texte veut suggérer que des déportés installés en Samarie viennent du sud de la Mésopotamie.

La deuxième partie montre comment le culte de YHWH continue dans le pays, suite à une invasion de lions envoyés par YHWH. Le roi d'Assyrie envoie alors chercher un prêtre déporté qui devient responsable du culte de YHWH à Béthel.

33. J.-D. Macchi, Les Samaritains. Histoire d'une légende. Israël et la province de Samarie, Genève, 1994, p. 59-66.

34. Voir la discussion chez V. Fritz, Das erste Buch der Könige, Zürich, 1996, p. 101.

35. R. ZADOK, «Geographical and Onomastic Notes », Journal of the Ancient Near Eastern Society 8 (1976), p. 113-126, spécialement p. 117. 
Malgré la vision négative de Béthel, le texte confirme que le sanctuaire de Béthel continua de jouer un rôle important après 722 avant notre ère. L'histoire du sanctuaire de Béthel est racontée avec une touche d'ironie: les interlocuteurs du roi d'Assyrie lui parlent «des nations que tu as déportées », celui-ci répond à ses hauts fonctionnaires d'aller chercher un des prêtres « que vous avez déportés », comme s'il ne voulait accepter la responsabilité de la déportation. De toute façon, le texte souligne la puissance de YHWH qui veille à la continuité de son culte.

L'épisode de l'invasion des lions reflète-t-il un événement historique - l'abandon ou le dépeuplement de certains sites pourrait être à l'origine d'une prolifération de lions -, ou est-elle une invention littéraire, le lion étant le totem de la tribu de Juda? Le motif peut aussi trouver son origine dans la reprise d'un traité assyrien d'Assarhaddon avec Baal, le roi de Tyr vers 676 avant notre ère. Dans ce traité, on annonce une invasion de lions comme une des punitions en cas de non-respect du contrat:

Que Béthel et Anat-Béthel vous livrent aux mâchoires d'un lion dévorant ${ }^{36}$.

Dans ce texte, Béthel apparaît comme une divinité, une sorte de matérialisation d'un bétyle. Ce traité est peut-être l'attestation la plus ancienne de cette divinitée ${ }^{37}$, une divinité vénérée en Phénicie, chez les Araméens. On trouve le nom au milieu de plusieurs noms propres des périodes néo-babylonienne et perse. Elle est également vénérée par des communautés araméennes en Égypte: une lettre trouvée à Hermopolis parle du temple de Béthel et du temple de la Reine du Ciel. À Eléphantine, on trouve la triade Ya'u, Ashim-bethel et Anat-bethel. Il est possible que Béthel ait aussi été vénéré comme un dieu en Israël, comme le suggère l'oracle de Jr 48,13: «Moab aura honte de Kamosh, tout comme la maison d'Israël a honte de Béthel, dans lequel elle se fiait ». La mise en parallèle de Béthel avec Kamosh parle en faveur du fait que ce nom désigne ici une divinité.

Mais revenons à $2 \mathrm{R} 17$ : ici Béthel désigne clairement le sanctuaire. Malgré l'importation d'autres divinités, dont certaines

36. Traduction selon J. BRIEnd, Traités et serments dans le ProcheOrient ancien, Paris, p. 66 (Supplément au Cahiers Évangile 81).

37. Pour plus de détails, voir W. Röllig, «Bethel», dans K. VAN DeR ToOrn - B. Becking - P. W. van Der Horst (éd.), Dictionary of Deities and Demons in the Bible, Leyde, 1999, p. 173-175. 
sont difficiles à identifier ${ }^{38}$, l'auteur de ce passage admet que le culte de YHWH continue en Samarie. Malheureusement, nous n'avons que très peu d'informations et souvent sous forme polémique, mais l'existence d'un sanctuaire yahwiste au Garizim confirme cette continuité. Ainsi l'étiologie du culte en Samarie après 722 avant notre ère reconnaît-elle l'importance du sanctuaire de Béthel et la continuité de la vénération du dieu YHWH. 2 R 17 se termine par le constat de la validité de cette vénération non orthodoxe de YHWH «jusqu'à ce jour», dépassant alors la notice de la destruction de Béthel en 2 R 23 et soulignant l'importance du sanctuaire et du culte yahwiste en «Samarie » à l'époque perse:

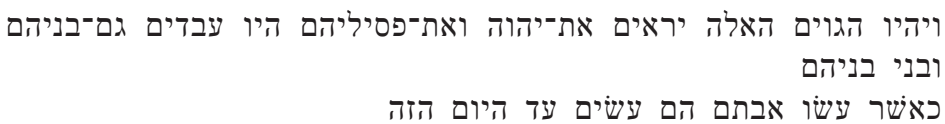

Ces nations craignaient YHWH et servaient leurs statues. Leurs fils et les fils de leurs fils agissent comme leurs pères ont agi jusqu'à ce jour. (2 R 17,41)

\section{L'idée du sanctuaire unique dans le contexte du Pentateuque}

Certains textes du Tétrateuque, notamment dans les livres de la Genèse et de l'Exode mettent en scène une diversité de sanctuaires yahwistes et ceci apparemment sans adopter une perspective critique. L'ouverture du Code d'alliance en Ex 20,24 semble ouvertement contredire Dt 12,14:

Tu me feras un autel de terre pour y sacrifier tes holocaustes et tes sacrifices de paix, ton petit et ton gros bétail; en tout lieu où je ferai rappeler mon nom, je viendrai vers toi et je te bénirai.

38. Certains dieux sont bien connus comme Nergal, dieu des enfers, qui a également des fonctions positives. Ashima est soit une parodie pour Ashérah, soit le «nom»divinisé (voir Am 8,14). Sukkot Banot n'est pas identifiable. On a postulé une déesse Banitu (M. Cogan, «Sukkoth-Benot », dans K. VAN DER TOORN - B. BECKING - P. W. VAN DER Horst (éd.), Dictionary of Deities and Demons in the Bible, Leyde, 1999, p. 821-822), mais avec J.-D. Macchi, on peut comprendre « huttes des filles $\gg$, une allusion à la prostitution. À noter que la liste commencerait avec des pratiques impliquant des jeunes filles et se terminerait par le sacrifice des fils dans le feu. 
L'explication traditionnelle et majoritaire consiste à comprendre Dt 12 comme une reprise d'Ex 20,24 dans le but de réécrire l'ancienne loi dans le sens de l'idéologie de la centralisation $^{39}$. John Van Seters et Christoph Levin, en revanche, ont proposé de voir en Ex 20,24 une critique récente de la loi de centralisation. L'insistance sur les différents lieux de culte depuis Noé jusqu'au Sinaï, en passant par les nombreux autels érigés par Abraham et Jacob, s'expliquerait selon C. Levin comme une polémique anti-deutéronomique. Le Yahwiste du début de l'époque perse rejetterait la théologie de la centralisation du culte ${ }^{40}$. Ex 20,24 pourrait alors se comprendre comme une réponse critique à Dt 12 soulignant la liberté totale de YHWH par rapport au lieu de son culte ${ }^{41}$. Il importe peu que l'on date l'introduction du Code d'alliance d'avant ou après Dt 12,13-18. Dans le contexte du Pentateuque, les deux conceptions cohabitent et, d'une certaine manière, Dt 12,14 se présente, sur le plan synchronique, comme une relecture d'Ex 20,24.

Les textes sacerdotaux du Pentateuque semblent être, eux aussi, moins intéressés à la centralisation absolue du culte sacrificiel. On suppose en général que Lv 1-7 et 17 présupposent la loi de centralisation de Dt 12. On peut cependant se demander si la conception sacerdotale d'un sanctuaire transportable au Sinaï ne signifie pas également de la part de $\mathrm{P}$ une certaine acceptation d'un sanctuaire yahwiste ailleurs qu'à Jérusalem, par exemple à Béthel ou à Samarie ${ }^{42}$. P serait alors moins préoccupé par la

39. Voir pars pro toto: B. M. Levinson, Deuteronomy and the Hermeneutics of Legal Innovation, New York-Oxford, 1997 (trad. française: L'herméneutique de l'innovation. Canon et exégèse dans l'Israël biblique, Bruxelles, 2005).

40. J. Van Seters, A Law Book for the Diaspora. Revision in the Study of the Covenant Code, Oxford, 2003; C. Levin, Der Jahwist, Göttingen, 1993, p. 430-432.

41. C. Levin, Der Jahwist, Göttingen, 1993, p. 431. Voir aussi J. Van Seters, A Law Book for the Diaspora. Revision in the Study of the Covenant Code, Oxford, 2003, p. 67: pour lui, l'enjeu de la loi de l'autel « is no longer of centralization of worship but one of religious survival... It allows for the simple construction of an altar in Jerusalem after the Temple's destruction and the continuation of the cult there or perhaps a temporary rudimentary altar in the diaspora $\gg$.

42. B.-J. Diebner, «Gottes Welt, Moses Zelt und das salomonische Heiligtum. Anmerkungen zur Vollendung des Tempels », dans T. RömeR (éd.), Lectio difficilior probabilior? L'exégèse comme expérience de décloison- 
centralisation que $\mathrm{D}$, et l'ouverture du code de sainteté en Lv 17,3-5 pourrait se lire comme une médiation entre Ex 20 et Dt 12.

Il est connu que le Pentateuque ne contient pas le mot Jérusalem, ce qui a permis d'interpréter le lieu choisi par $\mathrm{YHWH}$ (Dt 12) ou la montagne du sacrifice d'Abraham (Gn 22) comme désignant le Garizim ${ }^{43}$. Néanmoins la rencontre entre Abram et Melkisédek à Shalem (Gn 14,18), tout au début de l'histoire de peuple, contient une interprétation judéenne à peine voilée du lieu du vrai sanctuaire. Mais puisque la Torah n'est pas une affaire intra-judéenne, on peut déceler des traces qui renforcent une lecture «samaritaine» du lieu choisi par YHWH. C'est dans cette perspective qu'il faut comprendre l'ajout de Dt 27,4-26 ${ }^{44}$ ensemble avec 11,29-30 -, un des derniers textes du Deutéronome qui reprend partiellement le vocabulaire de Dt 12 (offrir les holocaustes, manger des sacrifices, être dans la joie $)^{45}$ en le transposant au Garizim ${ }^{46}$. Ainsi le Pentateuque se trouve-t-il encadré par les deux interprétations possibles du seul sanctuaire légitime, qui ont sans doute été renforcées dans un sens ou dans l'autre dans les manuscrits de la Torah gardés en Judée et en Samarie.

nement, Mélanges offerts à Françoise Smyth-Florentin, Heidelberg, 1991, p. 127-154.

43. Voir B.-J. Diebner, "Auf einem der Berge im Lande Morija" (Gen 22,2) oder: "In Jerusalem auf dem Berge Morija" (2Chr 3,1)?», Dielheimer Blätter zum Alten Testament 23-24 (1987), p. 174-179.

44. Sur l'ajout tardif de ce texte, voir N. NA'AmAN, «The Law of the Altar in Deuteronomy and the Cultic Site Near Shechem », dans S. L. McKenzie - T. Römer (éd.), Rethinking the Foundations. Historiography in the Ancient World and in the Bible. Essays in Honour of John Van Seters, Berlin/New York, 2000, p. 141-161.

45. Voir Dt 12,14.18 et 27,6-7.

46. Pour l'antériorité de Garizim en Dt 27,4, voir C. Nihan, «Garizim et Ébal dans le Pentateuque. Quelques remarques en marge de la publication d'un nouveau fragment du Deutéronome », Semitica 54 (2012), p. $185-210$. 


\section{Annexe}

\begin{tabular}{|c|c|c|}
\hline Ex 20,24 & Dt $12,13-14$ & Lv 17,3-5 \\
\hline $\begin{array}{l}\text { Tu me feras un autel } \\
\text { de terre pour y sacri- } \\
\text { fier tes holocaustes et } \\
\text { tes sacrifices de paix, } \\
\text { ton petit et ton gros } \\
\text { bétail; en tout lieu où } \\
\text { je ferai rappeler mon } \\
\text { nom, je viendrai vers } \\
\text { toi et je te bénirai. }\end{array}$ & $\begin{array}{l}\text { Garde-toi bien d'offrir } \\
\text { tes holocaustes dans } \\
\text { n'importe lequel des } \\
\text { lieux que tu verras; } \\
\text { c'est seulement au lieu } \\
\text { choisi par YHWH } \\
\text { dans une de tes tribus } \\
\text { que tu offriras tes ho- } \\
\text { locaustes; c'est là que } \\
\text { tu feras tout ce que je } \\
\text { t'ordonne. }\end{array}$ & $\begin{array}{l}\text { Si un homme de la } \\
\text { maison d'Israël égorge } \\
\text { un bœuf, un mouton } \\
\text { ou une chèvre dans } \\
\text { le camp ou même } \\
\text { l'égorge hors du camp } \\
\text { sans l'amener à l'entrée } \\
\text { de la tente de la ren- } \\
\text { contre pour l'apporter } \\
\text { en présent à YHWH, } \\
\text { devant la demeure de } \\
\text { YHWH, il répondra } \\
\text { du sang qu'il a versé: } \\
\text { cet homme-là sera } \\
\text { retranché du sein de } \\
\text { son peuple. } \\
\text { Ainsi les fils d'Israël } \\
\text { amèneront les ani- } \\
\text { maux qu'ils vou- } \\
\text { draient sacrifier en } \\
\text { pleine campagne; } \\
\text { ils les amèneront au } \\
\text { prêtre, à l'entrée de la } \\
\text { tente de la rencontre, } \\
\text { pour YHWH; ils les } \\
\text { sacrifieront à YHWH } \\
\text { à titre de sacrifice de } \\
\text { paix. }\end{array}$ \\
\hline
\end{tabular}

